\title{
Crack roughness in the two-dimensional random threshold beam model
}

\author{
Phani K. V. V. Nukala \\ Computer Science and Mathematics Division, Oak Ridge National Laboratory, Oak Ridge, Tennessee 37831-6164, USA \\ Stefano Zapperi \\ CNR-INFM, S3, Dipartimento di Fisica, Università di Modena e Reggio Emilia, Via G. Campi 213A, 41100 Modena, Italy \\ and ISI Foundation, Viale S. Severo 65, 10133 Torino, Italy \\ Mikko J. Alava \\ Department of Applied Physics, Helsinki University of Technology, FIN-02015 HUT, Finland \\ Srdan Šimunović \\ Computer Science and Mathematics Division, Oak Ridge National Laboratory, Oak Ridge, Tennessee 37831-6164, USA
}

(Received 14 April 2008; published 13 October 2008)

\begin{abstract}
We study the scaling of two-dimensional crack roughness using large scale beam lattice systems. Our results indicate that the crack roughness obtained using beam lattice systems does not exhibit anomalous scaling in sharp contrast to the simulation results obtained using scalar fuse lattices. The local and global roughness exponents ( $\zeta_{\text {loc }}$ and $\zeta$, respectively) are equal to each other, and the two-dimensional crack roughness exponent is estimated to be $\zeta_{\text {loc }}=\zeta=0.64 \pm 0.02$. Removal of overhangs (jumps) in the crack profiles eliminates even the minute differences between the local and global roughness exponents. Furthermore, removing these jumps in the crack profile completely eliminates the multiscaling observed in other studies. We find that the probability density distribution $p[\Delta h(l)]$ of the height differences $\Delta h(l)=[h(x+l)-h(x)]$ of the crack profile obtained after removing the jumps in the profiles follows a Gaussian distribution even for small window sizes $(l)$.
\end{abstract}

DOI: 10.1103/PhysRevE.78.046105

PACS number(s): 62.20.mm, 46.50.+a, 62.25.Mn

\section{INTRODUCTION}

Understanding the scaling properties of fracture surfaces still represents an unsolved problem despite two decades of intense research activities [1,2]. Experiments on several materials under different loading conditions have shown that the fracture surfaces are self-affine [3] and can be characterized by a roughness exponent $\zeta$. Experiments on several materials including metals [4], glass [5], rocks [6], and ceramics [7], have shown a universal out of plane roughness exponent of $\zeta \simeq 0.8$ for three-dimensional fracture surfaces irrespective of the material studied, as reviewed in Ref. [8]. Recent experimental evidence shows, however, that the picture is more complicated: such a scaling is valid at small and intermediate scales in the so-called fracture process zone (FPZ), while at large scales one observes a new regime with $\zeta \simeq 0.4$ attributed to crack line depinning [9-11]. Recent numerical investigations of the random fuse model indicate that the local roughness exponent in two dimensions does not depend on the size of the FPZ, but only on the fact that a FPZ is present or not [12].

In addition, it is by no means a priori clear that "simple" self-affinity is sufficient to describe the experiments. It has been argued that fracture surfaces may exhibit anomalous scaling [13]: the global exponent describing the scaling of the crack width with the sample size is larger than the local exponent measured on a single sample $[14,15]$. In this sense, it is necessary to introduce two roughness exponents a global exponent $(\zeta)$ and a local exponent $\left(\zeta_{\text {loc }}\right)$ to define the roughness of fracture surfaces. Anomalous scaling is noted in the numerical simulations as well $[16,17]$; however, its origin is not clear yet although in experiments it is conjectured to be an artifact of initial transient regime as the fracture front moves away from the initial notch [11]. Recent studies $[18,19]$ also suggest that the origin of anomalous scaling in numerical simulations in two dimensions may also be due to the existence of overhangs (jumps) in the crack profile, originating from crack branching. As a further complication, there is an on-going debate whether fracture surfaces exhibit multiaffine scaling [18-21], implying that one would observe a whole family of roughness exponents $\zeta_{q}$ depending on which statistical moment $q$ of the correlation function is measured.

The theoretical understanding of the origin and universality of crack surface roughness is often investigated by discrete lattice (fuse, central-force, and beam) models [2]. In these models the elastic medium is described by a network of discrete elements such as fuses, springs, and beams with random failure thresholds. In the simplest approximation of a scalar displacement, one recovers the random fuse model (RFM) where a lattice of fuses with random threshold are subject to an increasing external voltage [22]. Using twodimensional RFM, the estimated crack surface roughness exponents are: $\zeta=0.7 \pm 0.07 \quad[23], \zeta_{\text {loc }}=2 / 3 \quad[24]$, and $\zeta$ $=0.74 \pm 0.02[25]$. Recently, using large system sizes (up to $L=1024$ ) with extensive sample averaging, we found that the crack roughness exhibits anomalous scaling [26]. In particular, the local and global roughness exponents estimated using two different lattice topologies are $\zeta_{\text {loc }}=0.72 \pm 0.02$ and $\zeta$ $=0.84 \pm 0.03$. The reasons behind the origin of anomalous scaling in numerical simulations are not yet clear, although the existence of overhangs in the crack profile is expected to have contributed to anomalous scaling $[18,19]$. In comparison, the roughness exponents obtained from quasi twodimensional experiments, mainly obtained for paper 
samples, indicate a roughness exponent in the range $\zeta$ $\simeq 0.6-0.7 \quad[18,20,27-30]$, but occasionally significantly higher values have also been reported [31]. It is not known at this time whether this variation in $\zeta$ values is a reflection of practical difficulties in experimentally measuring $\zeta$-in paper it is difficult to have a scaling range spanning over several decades since the structure becomes three dimensional at small scales $(0.1 \mathrm{~mm})$ and at the millimeter range the fiber length interferes - or that the roughness exponent is not really universal but depends on material parameters and the anisotropy of the medium.

Despite this reasonable agreement between the numerical results obtained using two-dimensional RFM and the above quasi-two-dimensional experimental results, a lingering question is whether scalar representation of the elastic medium using random fuse models is an adequate representation of fracture. Moreover, it is an intriguing question whether the same roughness exponents as those obtained using RFM are found in more complex random threshold central-force (spring) and beam models. Using central-force models, a roughness exponent of $0.65 \pm 0.07$ is obtained in Ref. [32]. A more recent study with a range of disorder strengths in random thresholds estimated the local and global roughness exponents to be in the range 0.5-0.67 and 0.60.85 , respectively [19]. Using two-dimensional beam simulations, Ref. [33] estimated a typical value for the global roughness exponent to be 0.86 ; however, the roughness exponent is argued to be disorder dependent. Similar disorder dependent roughness exponents have also been reported recently for the RFM [34]. These results were obtained in the square lattice and were attributed to a lattice effect at low disorder. They indeed disappear for triangular lattice, where the roughness exponent is the same, independent of disorder [12].

The questions we address in this article are the following two: (i) whether anomalous scaling is present in twodimensional fracture simulations using beam lattice systems and (ii) what is the roughness exponent and what is its relation to those obtained using simplified scalar RFM models. Recent studies [18,20,29] have shown that multiaffinity of fracture surfaces is an artifact of overhangs (or jumps) in the crack profile. Here, we further investigate the influence of these overhangs in the crack profiles on crack roughness exponents. This article has three further Sections: in the next one, we describe the beam model used. In Sec. III, we present the numerical results obtained with it. Finally, Sec. IV concludes the paper.

\section{BEAM MODEL}

The random thresholds beam model (RBM) we consider in this study is a two-dimensional triangle lattice system of size $L \times L$. Unlike the scalar RFM model, the vectorial RBM has three degrees of freedom ( $x$ translation $u, y$ translation $v$, and a rotation $\theta$ about the $z$ axis) at each of the lattice nodes (sites), and each of the bonds (beams) in the lattice connects two nearest-neighbor nodes $[2,35,36]$. We assume that the beams are connected rigidly at each of the nodes such that the angle between any two beams connected at a node re-

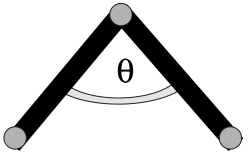

Undeformed

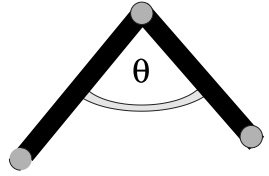

Stretching

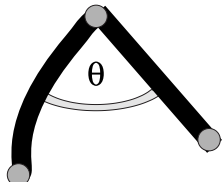

Bending
FIG. 1. Beams are connected rigidly at each of the nodes. The angle $\theta$ between two beams remains unaltered during the deformation that can be due to stretching or bending.

mains unaltered during the deformation process (see Fig. 1). These nodal displacements and rotations introduce conjugate forces and bending moments in the beam members.

In the RBM, we start with a fully intact lattice system with beams having unit length, unit square cross section, and Young's modulus $E=1$. This results in a unit axial stiffness $\left(E A / l_{b}=1\right)$ and bending stiffness $\left(12 E I / l_{b}^{3}=1\right)$ for each of the beams in the lattice system. Since the beam can deform in two independent deformation modes (axial and bending), we assume randomly distributed bond breaking axial and bending thresholds $t_{a}$ and $t_{b}$ based on thresholds probability distributions $p_{a}\left(t_{a}\right)$ and $p_{b}\left(t_{b}\right)$, respectively. The failure criterion for a beam is defined through an axial force and bending moment interaction equation (similar to the von Mises criterion in metal plasticity) given by

$$
r \equiv\left(\frac{F}{t_{a}}\right)^{2}+\frac{\max \left(\left|M_{i}\right|,\left|M_{j}\right|\right)}{t_{b}}=1 .
$$

The beam breaks irreversibly, whenever the failure criterion $r \geqslant 1$. The criterion used is the same as in Ref. [36], while one could in principle use other choices in the RBM (which has more degrees of freedom than scalar models, and offers thus other alternatives such as simple thresholds for axial and bending forces or the energy of the beam). Periodic boundary conditions are imposed in the horizontal direction and a constant unit displacement difference is applied between the top and the bottom of lattice system.

Numerically, a unit displacement, $\Delta=1$, is applied at the top of the lattice system and the equilibrium equations for force and momenta (see the appendix for details) are solved to determine the force in each of the beams, resulting in global displacements and rotations d. Using these values, local displacements $\mathbf{d}_{l}=\mathbf{T} \cdot \mathbf{d}$ and local forces $\mathbf{F}_{l}=\mathbf{K}_{\text {local }} \mathbf{d}_{l}$ are computed for each of the intact beams. Subsequently, for each bond $k$ with nodes $i$ and $j$, the quantities $a_{k}=\left(\frac{F}{t_{a}}\right)^{2}$ and $b_{k}=\frac{\max \left(\left|M_{i}\right|,\left|M_{j}\right|\right)}{t_{b}}$ are evaluated, and the bond $k_{c}$ having the smallest value

$$
r_{k}=\frac{-b_{k}+\sqrt{b_{k}^{2}+4 a_{k}}}{2 a_{k}}
$$

is irreversibly removed (when $a_{k}=0$, then $r_{k}=\frac{1}{b_{k}}$ ). The forces are redistributed instantaneously after a bond is broken implying that the stress relaxation in the lattice system is much faster than the breaking of a bond. Each time a bond is broken, it is necessary to re-equilibrate the lattice system in order to determine the subsequent breaking of a bond. The 


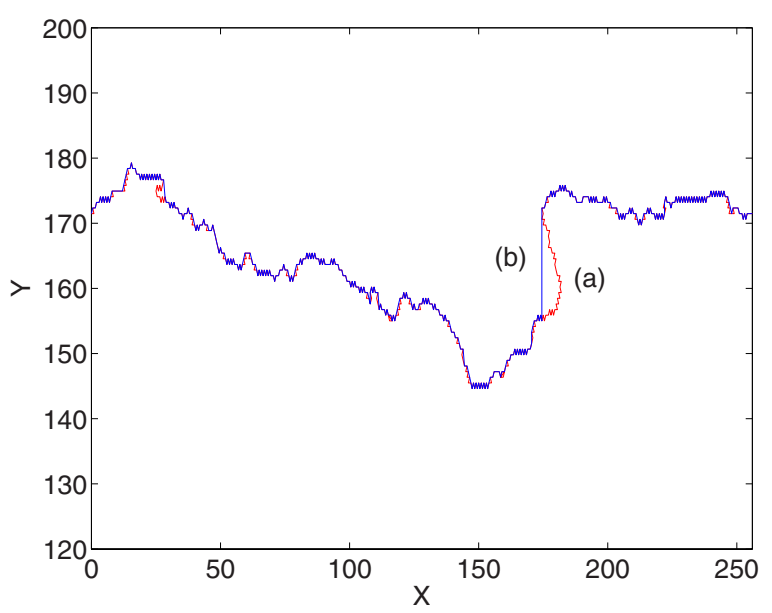

FIG. 2. (Color online) A typical crack in a system of size $L \times L$ with $L=256$. This crack, identified as (a) in the figure, has dangling ends, which are removed to obtain a single valued crack profile $h(x)$, identified as (b) in the figure. However, this final crack $h(x)$ possesses finite jumps that arise due to solid-on-solid projection of fracture surfaces.

process of breaking of a bond, one at a time, is repeated until the lattice system falls apart. For the RBM, we consider a uniform probability distribution in $[0,1]$ for both axial and bending thresholds disorders $P\left(t_{a}\right)$ and $P\left(t_{b}\right)$, respectively [i.e., $P(t)=1$ for $t$ in $[0,1]$ and zero otherwise].

Numerical simulations of fracture using lattice networks have often been limited to smaller system sizes due to the high computational cost associated with solving a new large set of linear equations every time a new lattice bond is broken. In this work, we use the multiple-rank sparse Cholesky factorization downdating algorithm developed by the authors for simulating fracture using discrete beam lattice systems $[37,38]$. For two-dimensional systems, this low-rank Cholesky factor downdating algorithm is significantly faster than competing preconditioned conjugate-gradient based iterative solvers. Using this numerical algorithm, we were able to investigate fracture in larger lattice systems (e.g., $L=320$ in 2D) than those investigated in previous studies. The lattice system sizes considered in this work are $L$ $=\{32,64,128,256,320\}$ with large numbers of sample configurations, $N_{\text {config }}=\{2000,4000,640,400,200\}$, respectively, in order to reduce the statistical error in the numerical results.

\section{CRACK ROUGHNESS}

Once the sample has failed, we identify the final crack, which typically displays dangling ends (see Fig. 2). We remove them and obtain a single valued crack line $h(x)$, where the values of $x \in[0, L]$ and $h(x)$ represents the local transverse position of the crackline at each $x$. For self-affine cracks, the local width $w(l) \equiv\left\langle\Sigma_{x}\left[h(x)-(1 / l) \Sigma_{X} h(X)\right]^{2}\right\rangle^{1 / 2}$, where the sums are restricted to regions of length $l$ and the average is over different realizations, scales as $w(l) \sim l^{\zeta}$ for $l \ll L$ and saturates to a value $W=w(L) \sim L^{\zeta}$ corresponding to the global width. The power spectrum $S(k) \equiv\left\langle\hat{h}_{k} \hat{h}_{-k}\right\rangle / L$,

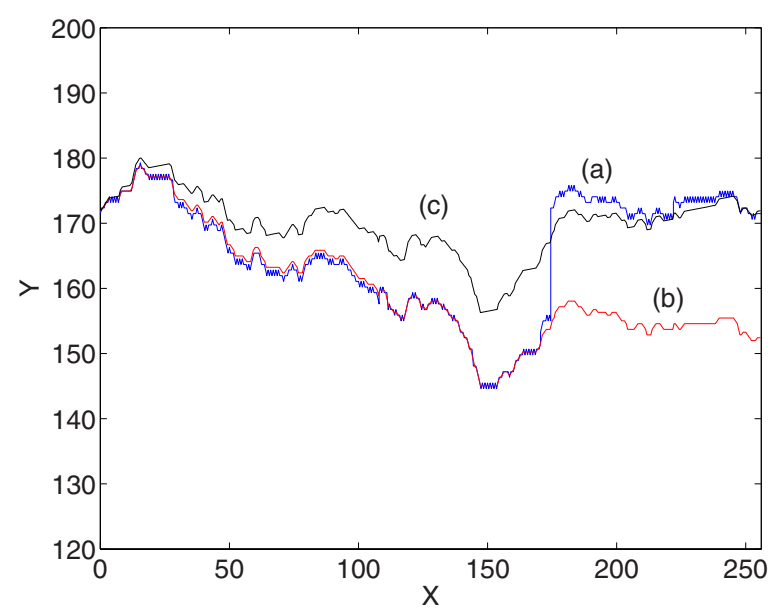

FIG. 3. (Color online) Figure shows a typical single valued crack profile $h(x)$ with jumps based on solid-on-solid projection scheme [identified as (a)]. Removing the jumps in the crack profile $h(x)$ makes it a nonperiodic profile [identified as (b)]. Subtracting a linear profile from this nonperiodic profile results in a periodic profile [identified as (c)].

where $\hat{h}_{k} \equiv \sum_{x} h(x) \exp i(2 \pi x k / L)$, decays as $S(k) \sim k^{-(2 \zeta+1)}$. When anomalous scaling is present [13-15], the exponent describing the system size dependence of the surface differs from the local exponent measured for a fixed system size $L$. In particular, the local width scales as $w(l) \sim l^{\zeta \text { loc }} L^{\zeta-\zeta_{\text {loc }} \text {, so }}$ that the global roughness $W$ scales as $L^{\zeta}$ with $\zeta>\zeta_{\text {loc }}$. Consequently, the power spectrum scales as $S(k)$ $\sim k^{-\left(2 \zeta_{\mathrm{loc}}+1\right)} L^{2\left(\zeta-\zeta_{\mathrm{loc}}\right)}$.

Recently, Bouchbinder et al. [21] have suggested that the crack line $h(x)$ is not self-affine; instead, it exhibits a much complicated multiaffine (or multiscaling) structure. This implies a nonconstant scaling exponent $\zeta_{q}$ for the $q$ th order correlation function $C_{q}(l)=\left\langle|h(x+l)-h(x)|^{q}\right\rangle^{1 / q} \sim l^{\zeta q} \quad[21]$. This would imply the breakdown of self-affinity. Recent studies $[18,20,29]$ have shown that multiaffinity of fracture surfaces seems to disappear on large enough scales $l$, however.

As shown in Fig. 3, removal of jumps from an initially periodic crack profile $h(x)$ makes the resulting crack profile $h_{\mathrm{NP}}(x)$ nonperiodic, where the subscript "NP" refers to nonperiodicity of the profiles. A direct evaluation of the roughness exponent using these nonperiodic profiles can be made. However, such an evaluation of roughness exhibits finite size effects for window sizes $l>L / 2$ due to nonperiodicity. Alternatively, the roughness of these resulting profiles can be evaluated by first subtracting a linear profile $h_{\text {lin }}(x)$ $=\left[h_{\mathrm{NP}}(0)+\frac{\left[h_{\mathrm{NP}}(L)-h_{\mathrm{NP}}(0)\right]}{L} x\right]$ from the nonperiodic profile $h_{\mathrm{NP}}(x)$, and then evaluating the roughness of the resulting periodic profile $h_{P}(x)$.

Figure 4(a) presents the scaling of crack width $w(l)$ with window size $l$. The inset and the main figure, respectively, show the crack widths calculated based on original crack profiles with jumps and those obtained from crack profiles without the jumps. The jumps in the profiles appear to result in slightly different local and global roughness exponents ( $\zeta_{\text {loc }}$ and $\zeta$, respectively), although the exponents are within error bars. However, removing these jumps in the crack pro- 

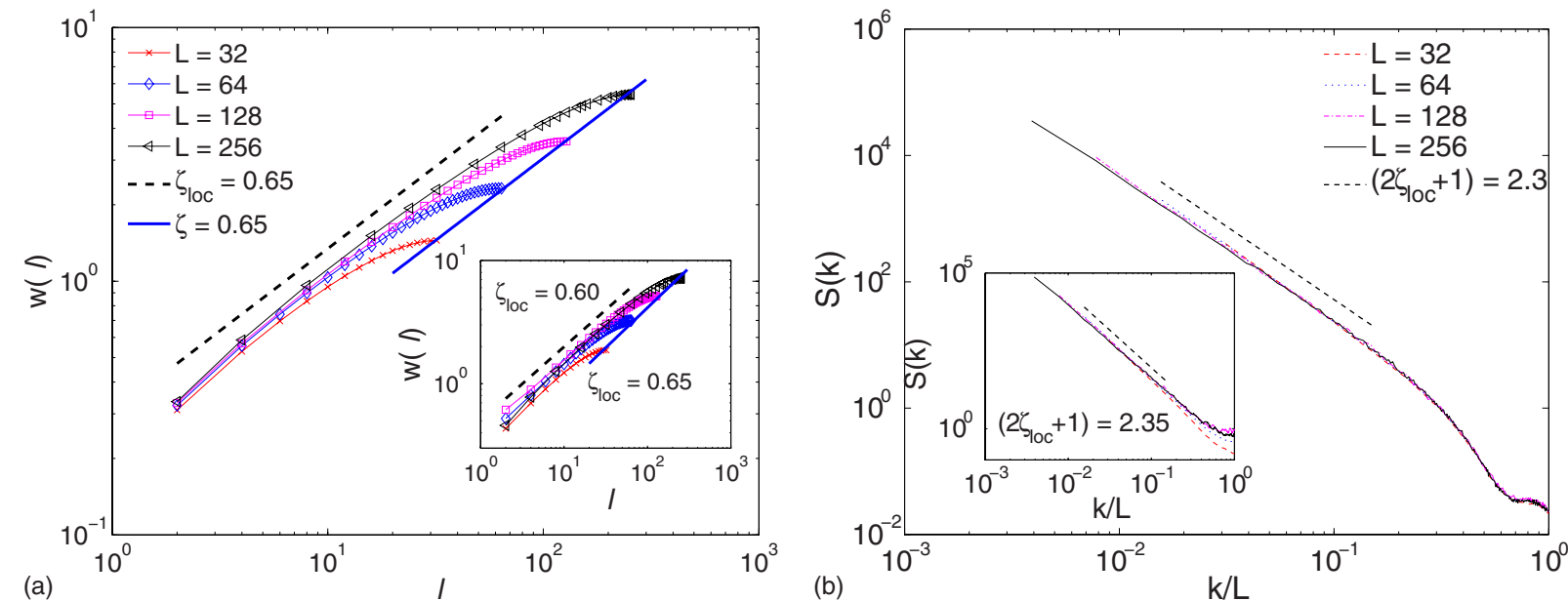

FIG. 4. (Color online) (a) Scaling of crack width $w(l)$ with window size $l$. The inset shows crack widths calculated based on original crack profiles with jumps. The local and global roughness exponents $\left(\zeta_{\text {loc }}\right.$ and $\zeta$, respectively) appear to be within error bars although the curves do not collapse. In the main figure, crack widths are calculated based on crack profiles obtained after removing the jumps in the profiles. Removing the jumps in the profiles appears to eliminate the discrepancy between local and global roughness exponents. (b) Scaling of power spectrum $S(k)$. Inset shows the scaling of $S(k)$ for original crack profiles whereas the main figure shows the scaling of $S(k)$ for crack profiles without jumps. Collapse of the power spectrum can be observed, and the exponents are consistent with those shown in (a).

files leads to a single roughness exponent of $\zeta_{\text {loc }}=\zeta=0.65$ suggesting that anomalous scaling is an artifact of jumps in the crack profiles, at least for fracture simulations based on beam lattice systems. We have also investigated the power spectra $S(k)$ of the crack profiles with and without the jumps in the crack profiles [see Fig. 4(b)]. Collapse of the power spectra for different system sizes can be observed for both the sets of crack profiles and the roughness exponents $\left(\zeta_{\text {loc }}\right.$ $=\zeta=0.65)$ obtained using the power law fits to the data are consistent with those presented in Fig. 4(a).

The self-affine property of the crack profiles also implies that the probability density distribution $p[\Delta h(l)]$ of the height differences $\Delta h(l)=[h(x+l)-h(x)]$ of the crack profile follows the relation

$$
p[\Delta h(l)] \sim\left\langle\Delta h^{2}(l)\right\rangle^{-1 / 2} f\left(\frac{\Delta h(l)}{\left\langle\Delta h^{2}(l)\right\rangle^{1 / 2}}\right) .
$$

Noting that periodicity in crack profiles is analogous to return-to-origin excursions arising in stochastic processes, we propose the following ansatz for the local width $\left\langle\Delta h^{2}(l)\right\rangle^{1 / 2}$ in height differences $\Delta h(l)$

$$
\left\langle\Delta h^{2}(l)\right\rangle^{1 / 2}=\left\langle\Delta h^{2}(L / 2)\right\rangle^{1 / 2} \phi\left(\frac{l}{L / 2}\right)
$$

with $\left\langle\Delta h^{2}(L / 2)\right\rangle^{1 / 2}=L^{\zeta}$. For periodic profiles, the function $\phi\left(\frac{l}{L / 2}\right)$ is symmetric about $l=L / 2$ and is constrained such that $\phi\left(\frac{l}{L / 2}\right)=0$ at $l=0$ and $l=L$, and $\phi\left(\frac{l}{L / 2}\right)=1$ at $l=L / 2$. Based on these conditions, a scaling ansatz of the form

$$
\left[\frac{\left\langle\Delta h^{2}(l)\right\rangle^{1 / 2}}{\left\langle\Delta h^{2}(L / 2)\right\rangle^{1 / 2}}\right]^{1 / \zeta_{\text {loc }}}+\frac{(l-L / 2)^{2}}{(L / 2)^{2}}=1
$$

similar to stochastic excursions or bridges can be proposed for $\left\langle\Delta h^{2}(l)\right\rangle^{1 / 2}$, which implies a functional form

$$
\phi\left(\frac{l}{L / 2}\right)=\left[1-\left(\frac{(l-L / 2)}{L / 2}\right)^{2}\right]^{\text {soc }}
$$

for $\phi\left(\frac{l}{L / 2}\right)$ that is satisfied to a good approximation by our numerical results. This scaling ansatz results in anomalous scaling when $\zeta_{\text {loc }} \neq \zeta$. Upon further simplification, Eq. (6) results in

$$
\phi\left(\frac{l}{L / 2}\right)=4_{\mathrm{loc}}^{\zeta_{\mathrm{loc}}}\left(\frac{l}{L}\right)^{\zeta_{\mathrm{loc}}}\left(1-\frac{l}{L}\right)^{\zeta_{\mathrm{loc}}}
$$

which along with $\left\langle\Delta h^{2}(L / 2)\right\rangle^{1 / 2}=L^{\zeta}$ and Eq. (4) shows how anomalous scaling arises and how local and global roughness exponents $\zeta_{\text {loc }}$ and $\zeta$ can be computed based on numerical results.

Figure 5 presents the scaling of $\left\langle\Delta h^{2}(l)\right\rangle^{1 / 2}$ based on the above ansatz [Eqs. (4) and (6)]. The collapse of the $\left\langle\Delta h_{P}^{2}(l)\right\rangle^{1 / 2} /\left\langle\Delta h_{P}^{2}(L / 2)\right\rangle^{1 / 2}$ data for different system sizes $L$ and window sizes $l$ onto a scaling form given by Eq. (6) with $\zeta_{\text {loc }}=0.64$ can be clearly seen in Fig. 5(a). In addition, the collapse of the data presented in Fig. 5(c) for $\left\langle\Delta h_{P}^{q}(l)\right\rangle^{1 / q} /\left\langle\Delta h_{P}^{q}(L / 2)\right\rangle^{1 / q}$ provides quite concrete evidence that multiaffine scaling of fracture surfaces, similar to what is observed in Ref. [21], is an artifact of jumps in the crack profile that are formed due to the solid-on-solid approximation used in extracting the crack profiles. The results in Fig. 5(c) clearly demonstrate that the removal of these jumps in the crack profiles completely eliminates this apparent multiscaling of fracture surfaces. This is also evident through the scaling of $\left\langle\Delta h_{P}^{q}(L / 2)\right\rangle^{1 / q}$ presented in Fig. 5(b). The slopes of the data for moments $q=1$ to 6 of $\Delta h_{P}(L / 2)$ are identical. It should also be noted that $\left\langle\Delta h_{P}^{q}(L / 2)\right\rangle^{1 / q} \sim L^{\zeta}$ with $\zeta=0.64$ and is identical to the local roughness exponent as obtained from Figs. 5(a) and 5(c). This further indicates that anomalous scaling of crack profiles is not present in fracture simulations obtained using the beam lattice systems. As already 

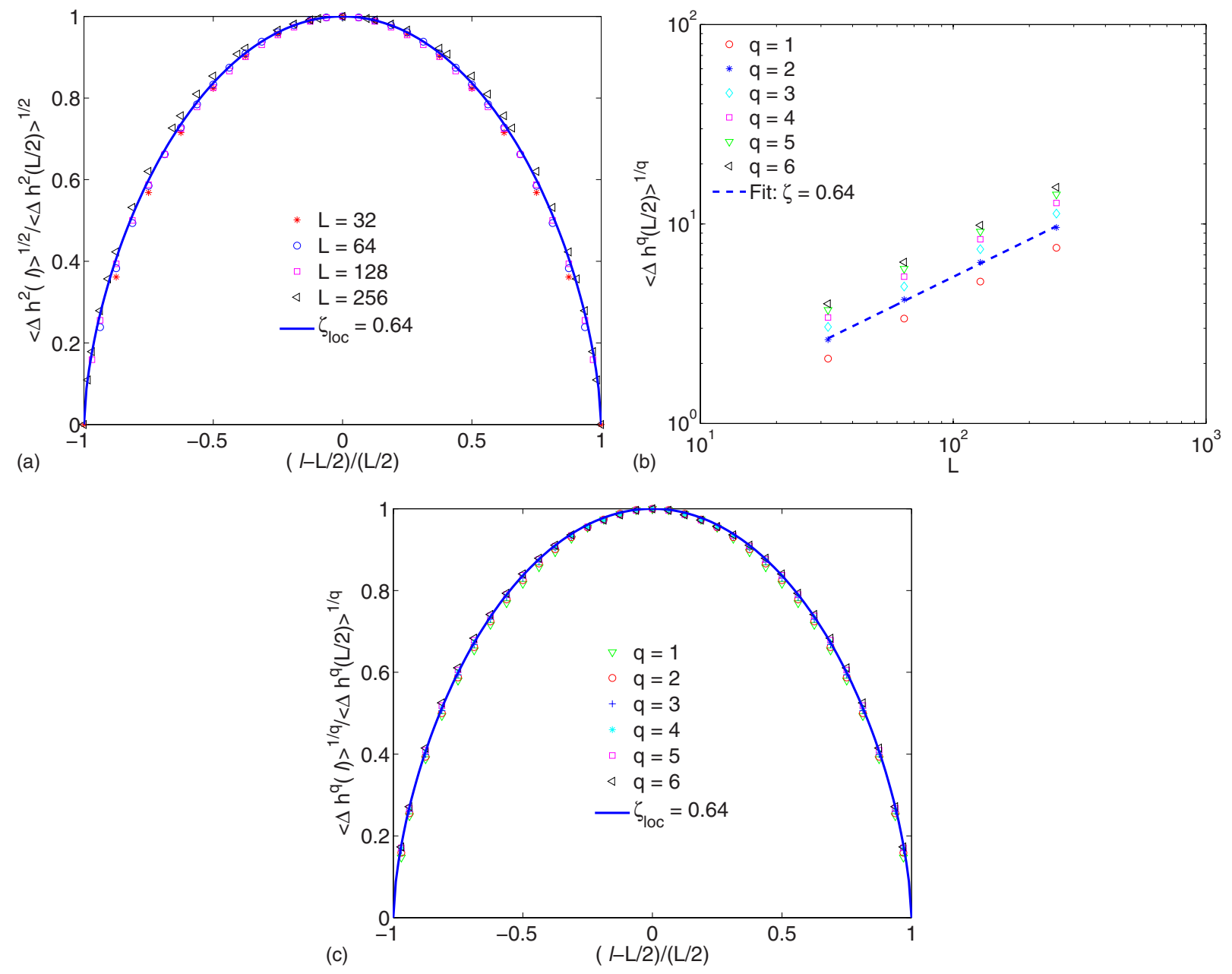

FIG. 5. (Color online) Removing the jumps from the crack profiles eliminates anomalous scaling. Furthermore, multiscaling of crack profiles is also due to the jumps in the crack profiles. (a) Scaling of $\left\langle\Delta h_{P}^{2}(l)\right\rangle^{1 / 2}$ with window size $l$ shows collapse of data onto Eq. (6) with $\zeta_{\text {loc }}=0.64$. (b) Scaling of $\left\langle\Delta h_{P}^{2}(L / 2)\right\rangle^{1 / 2}$ with system size $L$ with a scaling exponent of $\zeta=0.64$. (c) Scaling of $\left\langle\Delta h_{P}^{q}(l)\right\rangle^{1 / q}$ with window size $l$ shows that the apparent multiscaling of crack profiles is due to jumps in the profiles.

noted in Figs. 4(a) and 4(b), the difference between the local and global roughness exponents obtained using the original crack profiles is so small that it already negates the existence of anomalous scaling of crack roughness using beam lattice simulations. Removing the jumps caused by overhangs in the crack profile further reduces even this minute difference in local and global roughness exponents thereby eliminating anomalous scaling of crack profiles.

In the following, we investigate the probability density $p[\Delta h(l)]$ of height differences $\Delta h(l)$. In Refs. [18,20,29], the $p[\Delta h(l)]$ distribution is shown to follow a Gaussian distribution above a cutoff length scale and the deviations away from Gaussian distribution in the tails of the distribution have been attributed to finite jumps in the crack profiles. A selfaffine scaling of $p[\Delta h(l)]$ as given by Eq. (3) implies that the cumulative distribution $P[\Delta h(l)]$ scales as $P[\Delta h(l)]$ $\sim P\left[\Delta h(l) /\left\langle\Delta h^{2}(l)\right\rangle^{1 / 2}\right]$. Figure 6(a) presents the raw data of cumulative probability distributions $P[\Delta h(l)]$ of the height differences $\Delta h(l)$ on a normal or Gaussian paper for bin sizes $l \ll L$. As observed in Refs. [18,20,29], Fig. 6(a) shows large deviations away from Gaussian distribution for these small bin sizes. However, for moderate $l$, the distribution is Gauss- ian with deviations in the tails of the distribution beyond the $3 \sigma=3\left\langle\Delta h^{2}(l)\right\rangle^{1 / 2}$ limit (data not shown in figure). Removing the jumps in the crack profiles however collapses the $P\left[\Delta h_{P}(l)\right]$ distributions onto a straight line [see Fig. 6(b)] indicating the adequacy of Gaussian distribution even for small $l$. Indeed, Fig. 6(b) shows the collapse of the $P\left[\Delta h_{P}(l)\right]$ data for systems of sizes $L=128$ and $L=256$ with a variety of bin sizes $2 \leqslant l \leqslant L / 2$. Removing the jumps in the profiles not only turns the $P\left[\Delta h_{P}(l)\right]$ distributions Gaussian even for small window sizes $l$ but also extends the validity of $P\left[\Delta h_{P}(l)\right]$ Gaussian distribution for moderate bin sizes to a $4 \sigma=4\left\langle\Delta h_{P}^{2}(l)\right\rangle^{1 / 2}(99.993 \%$ confidence $)$ limit.

\section{DISCUSSION}

In summary, the analysis and results presented in this paper indicate that crack profiles obtained in fracture simulations using the beam lattice systems do not exhibit anomalous scaling of roughness and exhibit the local roughness exponent $\zeta_{\text {loc }}=0.65$. This has important indications since the RBM is the most faithful representation of the actual elasticity among all the lattice models discussed so far in the lit- 

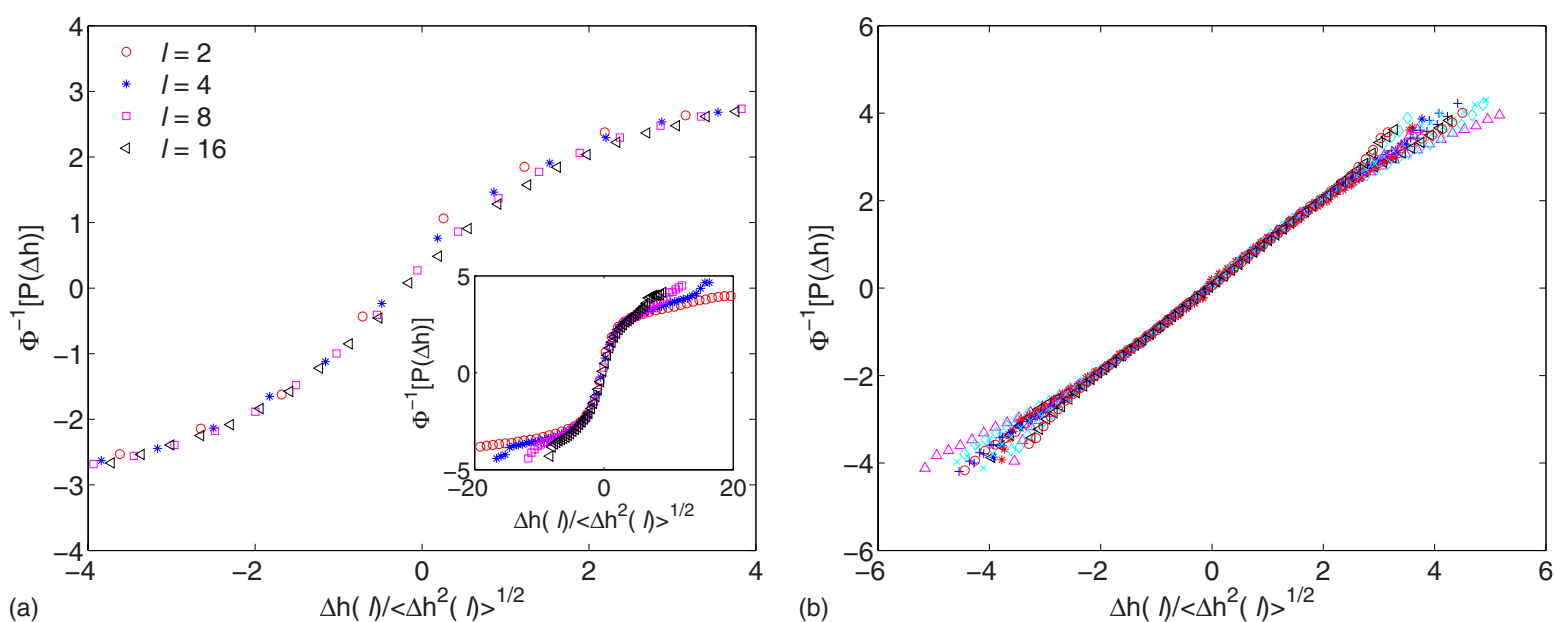

FIG. 6. (Color online) Normal paper plots of cumulative probability distributions $P[\Delta h(l)]$ of the height differences $\Delta h(l)=[h(x+l)$ $-h(x)$ ] of the crack profile $h(x)$ for various bin sizes $l . \Phi^{-1}$ denotes inverse Gaussian. The collapse of the profiles onto a straight line with unit slope would indicate that a Gaussian distribution is adequate to represent $P[\Delta h(l)]$. (a) $P[\Delta h(l)]$ distributions for $L=256$ and $l \ll L$. Large deviation from Gaussian profiles is observed for these window sizes. (b) Removing the jumps in the profiles, however, collapses the $P\left[\Delta h_{P}(l)\right]$ distributions onto a straight line indicating the adequacy of Gaussian distribution even for small window sizes $l$. The data is for $L=128$ with $l=(2,4,8,16,32,48,64)$, and $L=256$ with $l=(2,4,8,16,32,64,96,128)$. A total of 15 plots are shown in (b).

erature: an experiment in 2D fracture could be expected to result in similar scaling.

Comparing the result to other models (RFM, spring model) with signs of anomalous scaling, one sees nevertheless indications of similar local scaling: for the central-force spring model $\zeta_{\text {loc }}=0.65[32]$ and for the fuse model the relatively close value of $\zeta_{\text {loc }}=0.72$ [26]. Notice, however, that recently it was shown that for the random fuse model on the square lattice at low disorder the roughness exponent is larger [34]. This result was attributed to bias due to the lattice topology. In fact, for the triangular lattice this effect is not seen and the roughness exponent is universal [12]. The lattice geometry could also explain the difference between the present result for the beam model $\left(\zeta_{\text {loc }}=0.65\right)$ and previous disorder dependent results obtained for the square lattice [33].

The similarity in the local roughness exponent between the beam, spring, and possibly fuse models is interesting to note because of these model's dissimilarity in representing deformation of an elastic medium. This seems to imply that the anisotropy in the stress redistribution in tensorial models is irrelevant for the roughness, in two dimensions. We even obtain the same local roughness exponent by considering a simplified RBM in which failure events form a connected crack thereby excluding damage nucleation in the bulk. This simplified beam model is similar to the simplified random thresholds fuse model (RFM) considered in the Ref. [18]. In this model, after breaking the weakest beam, successive failure events are only allowed on beams that are connected to the crack while otherwise the rules are the usual RBM ones. Consequently, this model tracks only the connected crack along with its dangling ends in a disordered medium, and hence is the simples one for such studies.

As for the fuse models [18], this simplified beam model exhibits the same local roughness and power spectra characteristics as that of conventional RBM [see Fig. 7(a)]. This implies that one can expect to obtain same local roughness exponent as long as there exists a fracture process zone around the cracks. Additionally, Fig. 7(a) indicates the existence of anomalous scaling of roughness as soon as the branching of the cracks is allowed [39]. However, removing the jumps in the crack profiles eliminates this anomalous scaling as can be seen from the collapse of power spectra shown in Fig. 7(b). Thus it appears that the anomalous roughness in two-dimensional fracture simulations arises due to crack branching and coalescence of microcracks, which induce jumps in the crack profiles. It should also be noted that anomalous scaling of crack roughness is readily evident in fracture simulations obtained using fuse lattice systems. The reason for this greater propensity to exhibit anomalous scaling in fuse lattice systems appears to be due to scalar nature of fuse systems (antiplanar shear model), which readily allows for crack branching thereby inducing jumps in crack profiles.

\section{ACKNOWLEDGMENTS}

This research was sponsored by the Mathematical, Information and Computational Sciences Division, Office of Advanced Scientific Computing Research, U.S. Department of Energy under Contract No. DE-AC05-00OR22725 with UTBattelle, LLC. M.J.A. and S.Z. gratefully acknowledge the financial support of the European Commissions NEST Pathfinder program TRIGS under Contract No. NEST-2005PATH-COM-043386. M.J.A. also acknowledges the financial support from The Center of Excellence program of the Academy of Finland.

\section{APPENDIX: DETAILS ABOUT THE BEAM MODEL}

In the RBM model, beams are connected rigidly at each of the nodes and the angle between any two beams remains unaltered during the deformation process. These nodal 

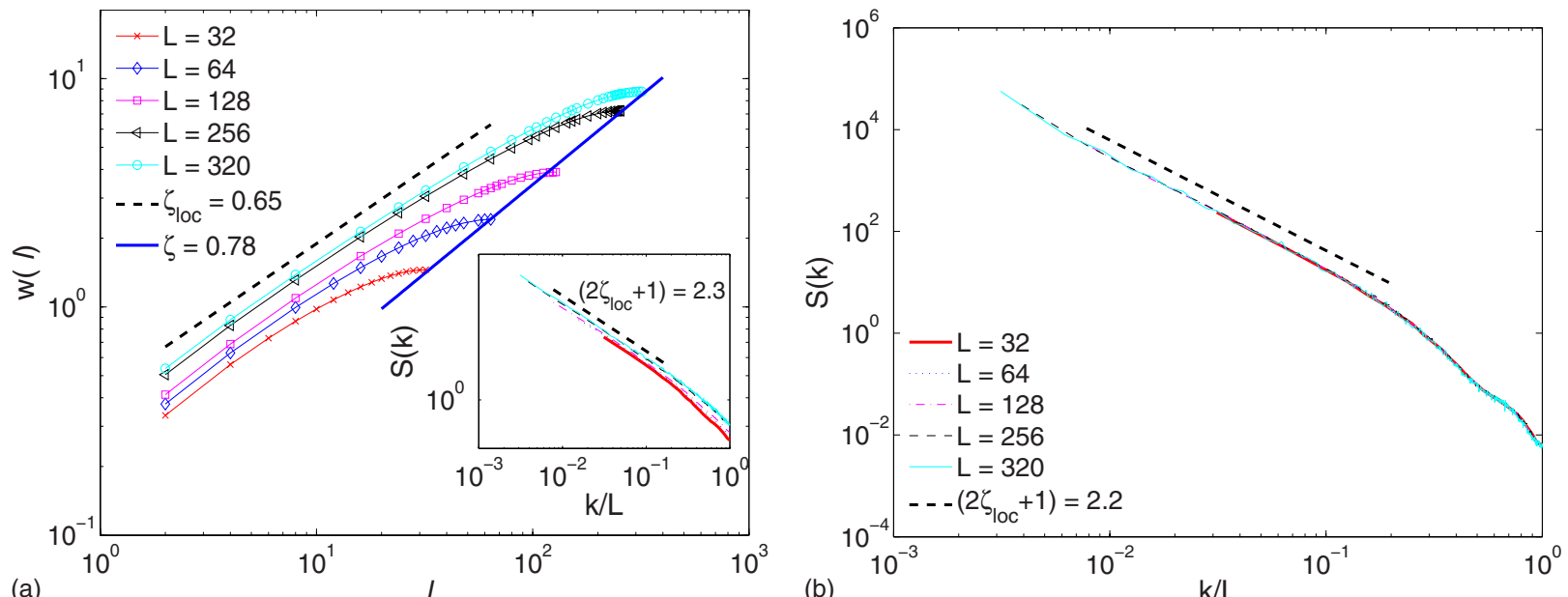

(b)

k/L

FIG. 7. (Color online) Scaling of crack widths and power spectra for the simplified RBM model. (a) Crack profiles (with jumps) seem to exhibit anomalous roughness scaling. However, the local roughness exponent is same as that noted in conventional RBM. The inset shows the scaling of power spectra, which are parallel to one another but do not collapse onto each other. (b) Collapse of power spectra of crack profiles obtained after removing the jumps in the profiles. Anomalous scaling of roughness in this simplified RBM appears to be due to these jumps in the profiles.

displacements and rotations introduce conjugate forces and bending moments. Using Timoshenko beam theory [40], which includes shear deformations of the beam cross section in addition to the usual axial deformation of cross sections, the local stiffness matrix for a beam element that relates the local nodal displacements and rotations to local nodal forces and bending moments in the beam's local coordinate system is given by

$$
\mathbf{K}_{\mathrm{local}}=\left[\begin{array}{cccccc}
\frac{E A}{l_{b}} & 0 & 0 & -\frac{E A}{l_{b}} & 0 & 0 \\
& \frac{12 E I}{(1+\alpha) l_{b}^{3}} & \frac{6 E I}{(1+\alpha) l_{b}^{2}} & 0 & -\frac{12 E I}{(1+\alpha) l_{b}^{3}} & \frac{6 E I}{(1+\alpha) l_{b}^{2}} \\
& \frac{(4+\alpha) E I}{(1+\alpha) l_{b}} & 0 & -\frac{6 E I}{(1+\alpha) l_{b}^{2}} & \frac{(2-\alpha) E I}{(1+\alpha) l_{b}} \\
& & \frac{E A}{l_{b}} & 0 & 0 \\
& & & \frac{12 E I}{(1+\alpha) l_{b}^{3}} & -\frac{6 E I}{(1+\alpha) l_{b}^{2}} \\
& & & & & \frac{(4+\alpha) E I}{(1+\alpha) l_{b}}
\end{array}\right],
$$

where $E$ is the Young's modulus, $G$ is the shear modulus, $A$ is the beam cross-sectional area, $I$ is the moment of inertia of beam cross section, $l_{b}$ is the length of the beam, and $\alpha=\frac{12 E I}{G A l_{b}^{2}}$ is the shear correction factor, which denotes the ratio of bending stiffness to the shear stiffness. If shear deformation of beam cross-section is negligible, then $\alpha=0$ and the Timoshenko's beam theory reduces to Euler-Bernoulli beam theory. Equation (A1) presents a relation between local nodal displacements and rotations $\mathbf{d}_{l}=\left(u_{l i}, v_{l i}, \theta_{l i}, u_{l j}, v_{l j}, \theta_{l j}\right)^{T}$ and local forces and moments $\mathbf{F}_{l}=\left(F_{l i}, V_{l i}, M_{l i}, F_{l j}, V_{l j}, M_{l j}\right)^{T}$. In this setting, the subscript $l$ refers to local quantities, the superscript $T$ represents transpose of a vector or a matrix, $i$ and $j$ refer to $i$ th and $j$ th nodes of the beam, and $F, V$, and $M$ refer to axial force, shear force, and bending moments, respectively.

Computing the equilibrium of the lattice system is achieved by first transforming these local quantities $\left(\mathbf{d}_{l}\right.$ and $\mathbf{F}_{l}$ ) into global quantities $\mathbf{d}=\left(u_{i}, v_{i}, \theta_{i}, u_{j}, v_{j}, \theta_{j}\right)^{T}$ and $\mathbf{F}$ $=\left(F_{i}, V_{i}, M_{i}, F_{j}, V_{j}, M_{j}\right)^{T}$ through a coordinate transformation 
$\mathbf{T}$ such that $\mathbf{d}_{l}=\mathbf{T} \cdot \mathbf{d}, \mathbf{F}_{l}=\mathbf{T} \cdot \mathbf{F}$, and $\mathbf{K}=\mathbf{T}^{T} \cdot \mathbf{K}_{\text {local }} \mathbf{T}$, and then satisfying equilibrium equations at each node such that

$$
\Sigma_{\langle i j\rangle} F_{x}=0 ; \quad \Sigma_{\langle i j\rangle} F_{y}=0 ; \quad \Sigma_{\langle i j\rangle} M=0
$$

where $\Sigma_{\langle i j\rangle}$ implies that the summation is carried over all the intact bonds $\langle i j\rangle$ joining at node $i$. In the above discussion, the transformation matrix $\mathbf{T}$ is given by

$$
\mathbf{T}=\left[\begin{array}{ll}
\mathbf{Q} & \mathbf{0} \\
\mathbf{0} & \mathbf{Q}
\end{array}\right],
$$

where

[1] H. J. Herrmann and S. Roux (eds.), Statistical Models for the Fracture of Disordered Media (North-Holland, Amsterdam, 1990).

[2] M. J. Alava, P. K. V. V. Nukala, and S. Zapperi, Adv. Phys. 55, 349 (2006).

[3] B. B. Mandelbrot, D. E. Passoja, and A. J. Paullay, Nature (London) 308, 721 (1984).

[4] K. J. Maloy, A. Hansen, E. L. Hinrichsen, and S. Roux, Phys. Rev. Lett. 68, 213 (1992); E. Bouchaud, G. Lapasset, J. Planés, and S. Navéos, Phys. Rev. B 48, 2917 (1993).

[5] P. Daguier, B. Nghiem, E. Bouchaud, and F. Creuzet, Phys. Rev. Lett. 78, 1062 (1997).

[6] J. Schmittbuhl, S. Roux, and Y. Berthaud, Europhys. Lett. 28, 585 (1994). J. Schmittbuhl, F. Schmitt, and C. Scholz, J. Fusion Energy 100, 5953 (1995).

[7] J. J. Mecholsky, D. E. Passoja, and K. S. Feinberg-Ringel, J. Am. Ceram. Soc. 72, 60 (1989).

[8] For a review see E. Bouchaud, J. Phys.: Condens. Matter 9, 4319 (1997). E. Bouchaud, Surf. Rev. Lett. 10, 73 (2003).

[9] L. Ponson, D. Bonamy, and E. Bouchaud, Phys. Rev. Lett. 96, 035506 (2006).

[10] D. Bonamy, L. Ponson, S. Prades, E. Bouchaud, and C. Guillot, Phys. Rev. Lett. 97, 135504 (2006).

[11] L. Ponson, D. Bonamy, H. Auradou, G. Mourot, S. Morel, E. Bouchaud, C. Guillot, and J. P. Hulin, Int. J. Fract. 140, 27 (2006).

[12] P. K. V. V. Nukala, S. Zapperi, M. J. Alava, and S. Simunovic, Phys. Rev. E 76, 056111 (2007).

[13] J. M. López, M. A. Rodríguez, and R. Cuerno, Phys. Rev. E 56, 3993 (1997).

[14] J. M. López and J. Schmittbuhl, Phys. Rev. E 57, 6405 (1998).

[15] S. Morel, J. Schmittbuhl, J. M. López, and G. Valentin, Phys. Rev. E 58, 6999 (1998).

[16] S. Zapperi, P. K. V. V. Nukala, and S. Simunovic, Phys. Rev. E 71, 026106 (2005).

[17] P. K.V. V. Nukala, S. Zapperi, and S. Simunovic, Phys. Rev. E 74, 026105 (2006).

[18] M. J. Alava, P. K. V. V. Nukala, and S. Zapperi, J. Stat. Mech.: Theory Exp. 2006, L10002 (2006).

[19] J. O. H. Bakke, T. Ramstad, and A. Hansen, Phys. Rev. B 76, 054110 (2007).

[20] S. Santucci, K. J. Maloy, A. Delaplace, J. Mathiesen, A. Hansen, J. O. H. Bakke, J. Schmittbuhl, L. Vanel, and P. Ray, Phys. Rev. E 75, 016104 (2007).

$$
\mathbf{Q}=\left[\begin{array}{ccc}
c & s & 0 \\
-s & c & 0 \\
0 & 0 & 1
\end{array}\right]
$$

and $c=\cos (\beta), s=\sin (\beta)$ refer to the direction cosines of the beam with $\beta$ representing the angle between the beam axis and the $x$ direction.
[21] E. Bouchbinder, I. Procaccia, S. Santucci and L. Vanel, Phys. Rev. Lett. 96, 055509 (2006) E. Bouchbinder, I. Procaccia, and S. Sela, J. Stat. Phys. 125, 1029 (2006).

[22] L. de Arcangelis, S. Redner, and H. J. Herrmann, J. Phys. (Paris), Lett. 46, 585 (1985).

[23] A. Hansen, E. L. Hinrichsen, and S. Roux, Phys. Rev. Lett. 66, 2476 (1991).

[24] E. T. Seppälä, V. I. Räisänen, and M. J. Alava, Phys. Rev. E 61, 6312 (2000).

[25] J. O. H. Bakke, J. Bjelland, T. Ramstad, T. Stranden, A Hansen, and J. Schmittbuhl, Phys. Scr. T106, 65 (2003).

[26] S. Zapperi, P. K. V. V. Nukala, and S. Simunovic, Phys. Rev. E 71, 026106 (2005).

[27] J. Kertész, V. K. Horvath, and F. Weber, Fractals 1, 67 (1993).

[28] T. Engoy, K. J. Maloy, A. Hansen, and S. Roux, Phys. Rev. Lett. 73, 834 (1994).

[29] L. I. Salminen, M. J. Alava, and K. J. Niskanen, Eur. Phys. J. B 32, 369 (2003).

[30] J. Rosti, L. I. Salminen, E. T. Seppälä, M. J. Alava, and K. J. Niskanen, Eur. Phys. J. B 19, 259 (2001).

[31] I. L. Menezes-Sobrinho, M. S. Couto, and I. R. B. Ribeiro, Phys. Rev. E 71, 066121 (2005).

[32] I. Malakhovsky, and M. A. J. Michels, Phys. Rev. B 74, 014206 (2006).

[33] B. Skjetne, T. Helle, and A. Hansen, Phys. Rev. Lett. 87, 125503 (2001).

[34] J. O. H. Bakke and A. Hansen, Phys. Rev. Lett. 100, 045501 (2008).

[35] S. Roux and E. Guyon, J. Phys. (Paris), Lett. 46, 999 (1985).

[36] H. J. Herrmann, A. Hansen, and S. Roux, Phys. Rev. B 39, 637 (1989).

[37] P. K. V. V. Nukala and S. Simunovic, J. Phys. A 36, 11403 (2003).

[38] P. K. V. V. Nukala, S. Simunovic, and M. N. Guddati, Int. J. Numer. Methods Eng. 62, 1982 (2005).

[39] As noted in Ref. [18] for the fuse models, when the branching of the cracks or damage within the fracture process zone is not allowed thereby limiting the crack extension to only the crack tips, a local roughness exponent of 0.5 is obtained and there is no anomalous scaling. As soon as branching is allowed in this simplified RBM model, the value of the roughness exponent increases from $\zeta_{\mathrm{loc}}=0.5$ to $\zeta_{\mathrm{loc}} \simeq 0.65$.

[40] J. S. Przemieniecki, Theory of Matrix Structural Analysis (McGraw-Hill, New York, 1968). 